\title{
Inverted papilloma of the ureter: study of a rare case with emphasis on clinicopathologic implications
}

\author{
Nikolaos Mertziotis, MD; ${ }^{*}$ Diomidis Kozyrakis, MD; ${ }^{*}$ Andreas Petrolekas, MD ${ }^{\dagger}$ Maria Terzi, MD; \\ Nikiforos Kapranos, $M D^{\ddagger}$
}

*Department of Urology, Mitera Clinic, Hygeia group, Marousi, Attica; 'Department of Urology, Henry Denaunt Hospital, Marousi, Attica; §Department of Pathology, Mitera Clinic, Hygeia group Marousi,

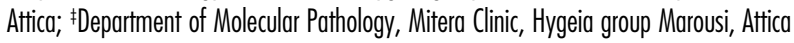

Cite as: Can Urol Assoc J 2012;6(6):E274-E276. http://dx.doi.org/10.5489/cuaj.11287

\section{Abstract}

A 62-year old male patient presented complaining of intermittent macroscopic hematuria. The ultrasonographic investigation revealed a hydronephrosis of remarkable degree with indiscrete renal parenchyma. The abdominal computed tomography scan identified a ureteral lesion with proximal dilatation, hydronephrosis and a functionless ipsilateral renal unit. The retrograde urography showed a 4-cm lesion with multiple filling defects and a smooth contour. The endoscopic examination showed an exophytic lesion, highly suspicious for malignancy. Urine cytology revealed atypia. Right nephroureterectomy was performed and the pathology revealed a ureteral inverted papilloma (UIP). Polymerase chain reaction examination for the presence of human papilloma virus, using GP5+/6+ consensus primers, was negative. The presence UIP should be considered in patients with urotheleal lesions in the ureter when the diagnostic workup for malignancy is inconclusive. The clinical course of the disease seems to be favorable.

\section{Introduction}

Inverted papilloma (IP) is a space occupying lesion encountered primarily in the nasal cavity and paranasal sinuses. ${ }^{1}$ It is a rare disease of the urinary tract. It has been detected, in order of frequency, in the bladder, the urethra and the ureter. ${ }^{2}$ It is considered a non-malignant disorder and its potential for recurrence is minimal. ${ }^{3,4}$ However, it is crucial to diagnose a synchronous or metachronous transition cell malignancy at presentation or during the follow- up respectively, to determines the treatment and disease prognosis., ${ }^{2,5}$ We present a rare case of IP detected in the collecting system of a functionless renal unit. The diagnostic workup, the pathology analysis and the prognostic factors are also discussed.

\section{Case report}

A 62-year-old male from a rural area of Eastern Greece, with no smoking history, presented to the urologic department with complaints of intermittent macroscopic hematuria and a dull right lumbar pain. The diagnostic workup included history taking, physical examination and imaging of the urinary tract. His symptoms started about 5 years ago, but at that time he ignored the recommendations for urologic consultation. He also suffered from severe kyphoscoliosis, which developed during his adolescence and from a recent onset of hypertension, which was resistant to medication. He took enalapril maleate $20 \mathrm{mg}$ and amlodipine $10 \mathrm{mg}$ once daily.

The ultrasonographic investigation revealed a right highgrade hydronephrosis with indiscrete renal parenchyma. The abdominal computed tomography scan (CT) identified an ipsilateral lesion located in the upper ureter with proximal dilatation, hydronephrosis and a functionless renal unit (Fig. 1 ). The retrograde urography showed a 4-cm lesion with multiple filling defects. Urine cytology revealed atypia, but malignant cells were not detected. In ureteroscopy, an exophytic ureteral mass was detected and it was highly suspicious for ureteral malignancy. Open right nephroureterectomy was performed and both the frozen section and the formalin- fixed paraffin- embedded tissue examination revealed a typical ureteral IP (Fig. 2). Urothelial carcinoma was not diagnosed. A polymerase chain reaction examination for the presence of human papilloma virus (HPV), using GP5+/6+ consensus primers, was negative. Not surprisingly, the postsurgical blood pressure measurements returned to quasi normal levels (<140/90 $\mathrm{mmHg}$ ) without medication. The follow-up protocol included urinalysis, total blood count and renal biochemistry every 3 months; cystoscopy and urine cytology every 6 months, and urinary tract imaging every year. Fourteen months after treatment, the patient has a satisfactory health status, with normal renal function, and no signs of tumour recurrence. 


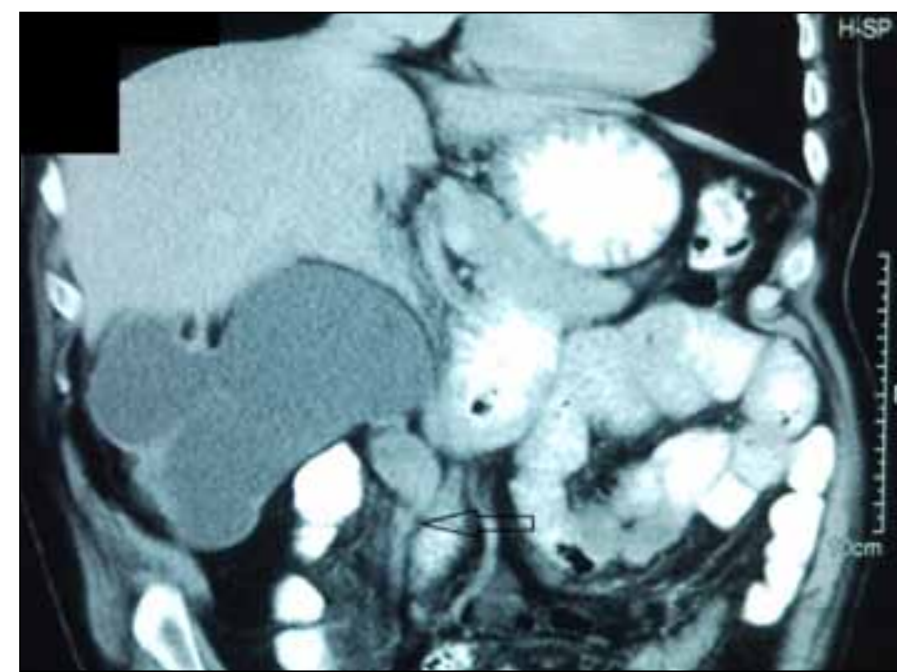

Fig. 1. A computed tomography image (coronal reconstruction) showing a ureteral lesion detected in the upper ureter (arrow) with severe hydronephrosis and a degenerated ipsilateral renal parenchyma.

\section{Discussion}

The first description of urothelial IP was published by Potts and Hirst in the early $70 \mathrm{~s},{ }^{6}$ or even earlier by Paschkis in 1927.7 Paschkis described the "adenoma-like tumours" of the bladder which, according to some authors, ${ }^{8,9}$ is identical to the those described by Potts and Hirst. IP is a rare space occupying lesion of the urinary tract ( $2 \%$ of all tumours) and its primary site is the bladder (90\% of urothelial IP). ${ }^{9,10}$ In a series of 41 lesions, $3(7 \%)$ were in the ureter. ${ }^{2}$ IP of the upper urinary tract is very rare and, reviewing the international literature, it has been described in no more than 50 cases. $^{2-4,9-13}$
The typical histologic pattern of the lesion comprises an endophytic trabeculation of the urothelial layer with downward development up to the stoma lining without any exophytic papillary component. ${ }^{1,14}$ Generally speaking, IP is a benign disorder, but in some specimens atypic features may be recognized, including the detection of prominent nuclei, atypical squamous characteristics, urothelial dysplasia and giant multinucleated cells. ${ }^{14} \mathrm{~A}$ lesion with atypia is of clinical importance because it is difficult to be distinguished from transitional cell carcinoma (TCC) with inverted growth pattern, has high probability of recurrence and has been associated with a previous, simultaneous or subsequent malignancy, thereby justifying a rigorous follow-up protocol. ${ }^{1,5,14,15}$

Generally speaking, the HPV virus is implicated in the development of up to $25 \%$ of the nasal and paranasal IP lesions. Viral genotypes ${ }^{16,17}$ that are high risk for malignancy have been associated with frequent recurrences and atypia of IP. ${ }^{16}$ Some authors documented the association of this virus with IP lesions located in the bladder (up to $87.5 \%$ detection rate) and the markers for high-risk genotypes were higher in atypical cases. ${ }^{18}$ Others failed to confirm this correlation and the role of HPV in urothelial IP is open to further study. ${ }^{17}$ We examined the surgical specimen of our patient for the presence of the virus, but we were not able to detect a HPV genome. To our knowledge, examination for the presence of the virus in ureteral lesions has never been performed.

In the absence of concomitant malignancy, local excision of the disease has been performed endoscopically ${ }^{10}$ or laparoscopicaly ${ }^{13}$ or as an open procedure. ${ }^{4}$ With a follow-up of 3.5 years after minimal invasive excision, no recurrence was reported. ${ }^{10}$ When the diagnostic workup was considered inconclusive, more aggressive treatment was proposed..$^{4,11}$

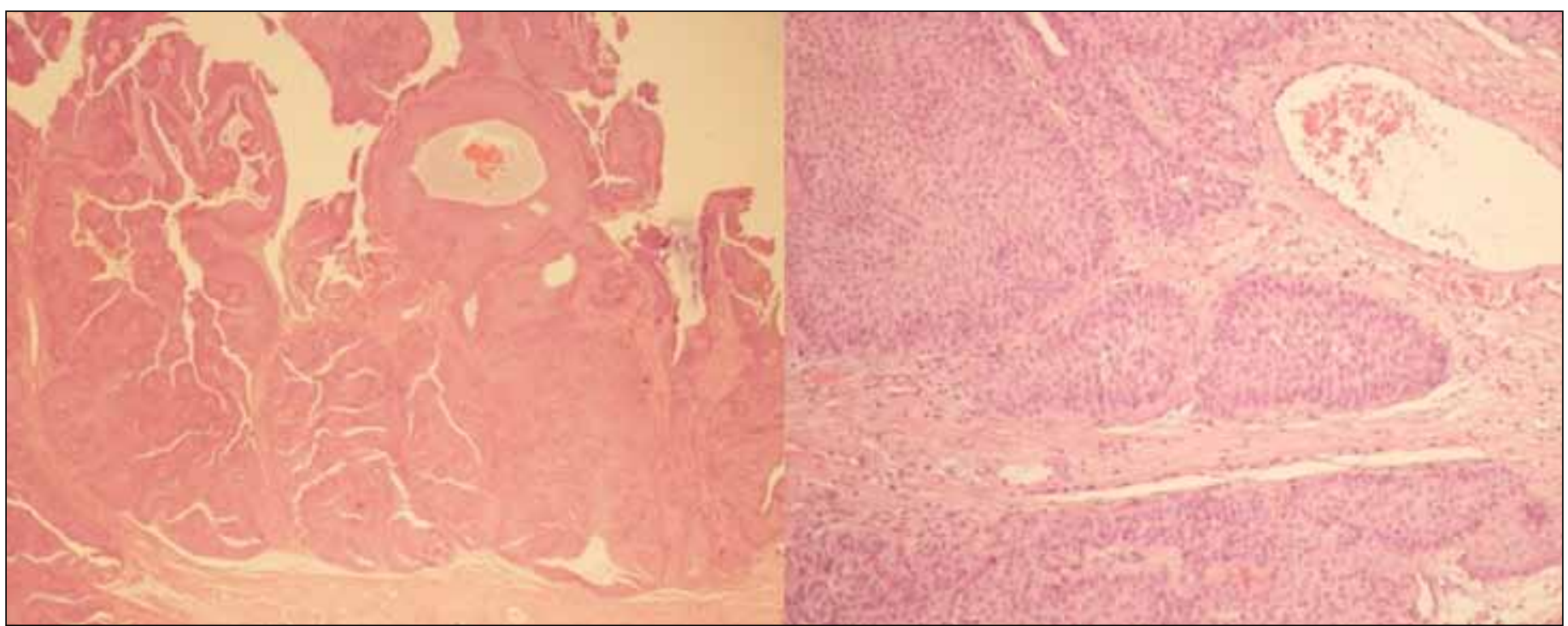

Fig. 2. Microphotograph of the ureteral IP. A. Endophytic non-invasive appearance of the tumor (haematoxylin and eosin; original magnification $\times 100)$. B. Cords and trabeculae of urothelial cells growing into underlying lamina propria (haematoxylin and eosin; original magnification $\times 200$ ). 
The ureteral lesion of our case co-existed with a nonfunctioning renal unit and was associated with a difficult to treat arterial hypertension. The endoscopic appearance of the lesion along with the images of both the abdominal CT and the urography, despite the negative cytology, could be attributed to a malignant urothelial lesion; consequently, radical intervention was performed. To our opinion, a more accurate preoperative diagnosis was difficult to make. Even if an IP had been diagnosed preoperatively, with biopsy samples taken under endoscopic guidance, the presence of a concomitant TCC could not be ruled out that time. Consequently, a formalin fixed paraffin embedded biopsy from the ureteral lesion was not performed preoperatively because the results would not change our therapeutic strategy. The kidney was functionless and had to be removed because of the presence of complications (nephrogenic hypertension) and the risk for others (infection, urosepsis, hematuria etc.), irrespective of the accurate diagnosis of the ureteral lesion. We believe that since nephrectomy was unavoidable, we were not justified to leave behind the coexisted ureteral lesion regardless of the results of the biopsy. A functionless renal unit has been described by other authors; they also emphasized the need for nephrectomy in such cases. ${ }^{12}$

The differential diagnosis may be sometimes challenging and includes primarily the urothelial (transitional cell) carcinoma and rarely the nephrogenic adenoma, the paraganglioma and the carcinoid tumours. ${ }^{2}$

In our patient, the pathologic diagnosis of a typical IP and the absence of TCC sites, along with a negative HPV investigation, justified a non-strict follow-up protocol with, as indicated by others, endoscopic examinations twice a year. ${ }^{2}$ Postoperatively, non-invasive and inexpensive tests were performed to detect hematuria, papilloma recurrences, metachronous urothelial tumour or renal deficiency.

Limitations of the paper include the retrograde nature of the study and the short follow-up period. Given that this is a solitary case, our findings and conclusions should be interpreted and generalized with caution.

\section{Conclusions}

Despite its rarity, the presence of inverted IP should be considered in patients with urotheleal lesions located in the ureter when the diagnostic workup for malignancy is inconclusive. A strict follow-up protocol with frequent inva- sive examinations of the urinary tract is not justified in the absence of atypical IP or urothelial malignancies.

Competing interests: None declared.

This paper has been peer-reviewed.

\section{References}

1. Sandison A. Common head and neck cases in our consultation referrals: diagnostic dilemmas in inverted papilloma. Head Neck Pathol 2009;3:260-2. http://dx.doi.org/10.1007/s12105-009-0136-z

2. Brown AL, Cohen RJ. Inverted papilloma of the urinary tract. BJU Int 2011;107 (Suppl 3):24-6. http:// dx.doi.org/10.1111/i.1464-410X.2011.10046.x

3. Sung MT, Maclennan GT, Lopez-Beltran A, et al. Natural history of urothelial inverted papilloma. Cancer 2006;107:2622-7. http://dx.doi.org/10.1002/cncr.22311

4. Asano K, Miki J, Maeda S, et al. Clinical studies on inverted papilloma of the urinary tract: report of 48 cases and review of the literature. J Urol 2003;170(4 Pt 1):1209-12. http://dx.doi.org/10.1097/01. ju.0000085342.15918.d7

5. Ho H, Chen YD, Tan PH, et al. Inverted papilloma of urinary bladder: is long-term cystoscopic surveillance needed? A single center's experience. Urology 2006;68:333-6. http://dx.doi.org/10.1016/j. urology.2006.03.014

6. Potts IF, Hirst E. Inverted papilloma of the bladder. J Urol 1963;90:175-9.

7. Paschkis R. Über adenome der harnblase. Z Urol Chir 1927;21:315.

8. Anderstrom C, Johansson S, Pettersson S. Inverted papilloma of the urinary tract. J Urol 1982;127:1132-4.

9. Kyriakos M, Royce R. A Case Report with a review of ureteral and renal pelvic inverted papillomas. Cancer 1989;63:368-80. http://dx.doi.org/10.1002/1097-0142(19890115)63:2<368::AIDCNCR2820630229>3.0.C0;2-G

10. Spevack L, Herschorn S, Srigley J. Inverted papilloma of the upper urinary tract. J Urol 1995;153:1202-4. http://dx.doi.org/10.1016/S0022-5347(01)67552-9

11. Schulze S, Holm-Nielsen A, Ravn V. Inverted papilloma of upper urinary tract. Urology 1986;28:58-61. http://dx.doi.org/10.1016/0090-4295(86)90186-X

12. Ogata $M$, Konari $S$, Abe $T$, Komatsu $S$, et al. Inverted papilloma of the ureter with non-functional kidney: A case report [in Japanese]. Hinyokika Kiyo 2003;49:741-3.

13. Lyon MB, Zorn KC, Orvieto MA, et al. Case Report: Laparoscopic Resection of Ureteral Inverted Papilloma. J Endourol 2006;20:399-401. http://dx.doi.org/10.1089/end.2006.20.399

14. Broussard JN, Tan PH, Epstein Jl. Atypia in inverted urothelial papillomas: pathology and prognostic significance. Hum Pathol 2004;35:1499-504. http://dx.doi.org/10.1016/i.humpath.2004.09.010

15. Cheng $\mathrm{CW}$, Chan $\mathrm{LW}$, Chan $\mathrm{CK}$, et al. Is surveillance necessary for inverted papilloma in the urinary bladder and urethra? ANZ I Surg 2005;75:213-7. http://dx.doi.org/10.1111/i.1445-2197.2005.03327.x

16. Lawson W, Schlecht NF, Brandwein-Gensler M. The role of the human papillomavirus in the pathogenesis of Schneiderian inverted papillomas: an analytic overview of the evidence. Head Neck Pathol 2008;2:4959. http://dx.doi.org/10.1007/s12105-008-0048-3

17. Gould VE, Schmitt M, Vinokurova $S$, et al. Human papillomavirus and $p 16$ expression in inverted papillomas of the urinary bladder. Cancer Let 2010;292:171-5. http://dx.doi.org/10.1016/i.canlet.2009.11.022

18. Shigehara K, Sasagawa T, Doorbar J, et al. Etiological role of human papillomavirus infection for inverted papilloma of the bladder. J Med Virol 2011;83:277-85. http://dx.doi.org/10.1002/imv.21966

Correspondence: Dr. Diomidis Kozyrakis, Erithrou Stavrou 6 Str., 15123, Marousi, Attica; fax: 00302106840894; dkoziraki@@yahoo.gr 